\title{
\begin{tabular}{l|} 
EXPERT \\
REVIEWS
\end{tabular}
}

\section{Plasmodium falciparum: new molecular targets with potential for antimalarial drug development}

Expert Rev. Anti Infect. Ther. 7(9), 1087-1098 (2009)

Donald L Gardiner', Tina S Skinner-Adams, Christopher L Brown, Katherine T Andrews, Colin M Stack, James S McCarthy, John P Dalton and Katharine R Trenholme ${ }^{+}$Author for correspondence Malaria Biology Laboratory, Queensland Institute of Medical Research, 300 Herston Road, Herston, QLD 4006, Australia Tel.: +61733620432 Fax: +61 733620104 don.gardiner@qimr.edu.au

Malaria remains one of the world's most devastating infectious diseases. Drug resistance to all classes of antimalarial agents has now been observed, highlighting the need for new agents that act against novel parasite targets. The complete sequencing of the Plasmodium falciparum genome has allowed the identification of new molecular targets within the parasite that may be amenable to chemotherapeutic intervention. In this review, we investigate four possible targets for the future development of new classes of antimalarial agents. These targets include histone deacetylase, the aspartic proteases or plasmepsins, aminopeptidases and the purine salvage enzyme hypoxanthine-xanthine-guanine phosphoribosyltransferase.

KEYwORDS: aminopeptidase $\bullet$ aspartic protease $\bullet$ histone deacetylase $\bullet$ hypoxanthine-xanthine-guanine phosphoribosyltransferase $\bullet$ malaria $\bullet$ molecular target $\bullet$ plasmepsin $\bullet$ Plasmodium falciparum

Parasites of the genus Plasmodium are the causative agents of malaria, man's most lethal parasitic disease. Each year, millions of people in tropical and subtropical regions of the world are infected with malaria parasites and approximately 1 million of these infections result in death $[1,101]$. While four Plasmodium species commonly infect humans, the two that cause the most morbidity and mortality are Plasmodium falciparum and Plasmodium vivax. $P$. falciparum is the cause of most deaths, which occur mainly in children and pregnant women in sub-Saharan Africa [2,3]. P. vivax, although it causes relatively fewer deaths, is responsible for significant morbidity, particularly in South America and the Asia-Pacific region [4].

Much of the current antimalarial pharmacopeia was identified serendipitously and, unlike current target-based drug discovery programs, the mode of action of these agents is still poorly understood. Quinine, the first widely used antimalarial agent, derived from the bark of the Cinchona tree and first isolated in $1820[5,6]$, and now artemisinin (also known as qinghaosu), an extract of sweet wormwood (Artemisia annua) [6,7], are derived from long-established herbal remedies from South America and China, respectively. Many of the antimalarial drugs in common use today are structural derivatives of these agents.
Despite the availability of effective antimalarial drugs, the prevention and treatment of malaria is progressively becoming more difficult due to the global spread of drug resistance. Almost all $P$. falciparum parasites are resistant to chloroquine [8], and other affordable antimalarial drugs such as sulfadoxine/pyrimethamine are becoming less effective $[9,10]$. The recent emergence of tolerance to the artemisinin drugs [11,12] is also of great concern and highlights the ongoing need for drug development. Thus, new antimalarial agents that act against novel parasite targets are required to combat multidrug-resistant parasites.

Malaria parasites have a complex life cycle and there are a number of key stages that are targeted by current antimalarial drugs and are potential targets for new drugs. The life cycle of $P$. falciparum is shown in Figure 1. Infection in humans begins when an infected female anopheles mosquito feeds and injects sporozoites into the host's bloodstream. These sporozoites rapidly invade liver cells where they multiply extensively and form exoerythrocytic schizonts, each containing up to 30,000 merozoites. A total of 6-16 days after infection (depending on the species), the schizont-infected hepatocytes rupture, releasing mature merozoites into the bloodstream. These 


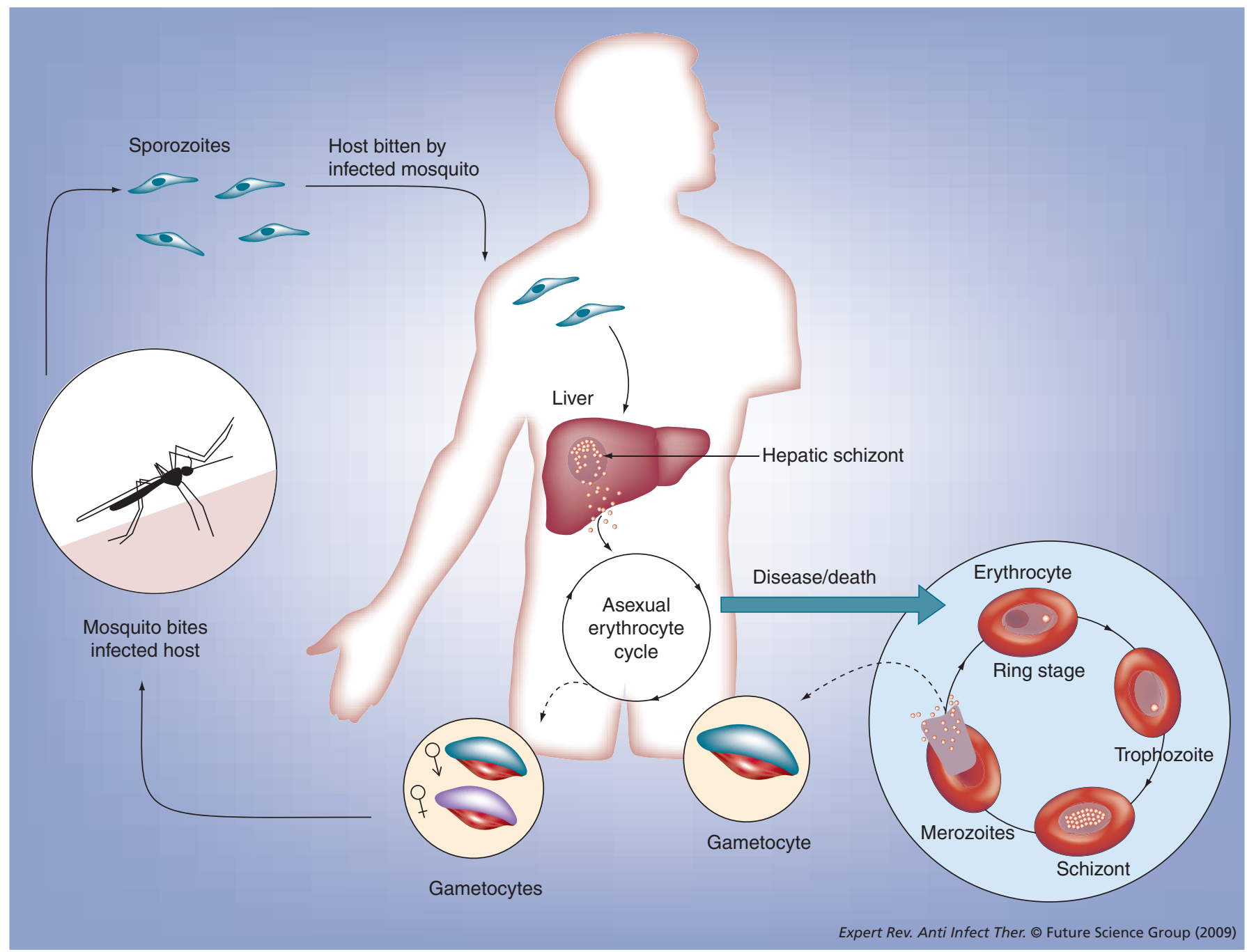

Figure 1. Life cycle of the human malaria parasite Plasmodium falciparum.

merozoites invade red blood cells (RBCs) and undergo a second round of multiplication that lasts $48-72 \mathrm{~h}$ and produces up to 32 merozoites. The released merozoites invade new RBCs and continue the asexual replication cycle.

The asexual erythrocytic lifecycle of $P$. falciparum is relatively synchronous in the natural host, lasting for $48 \mathrm{~h}$. In synchronous infections, the rupture of the infected RBCs and merozoite release are associated with the characteristic fever and acute symptoms of malaria. Some merozoites also give rise to sexually differentiated forms (gametocytes). The trigger for gametocytogenesis is unclear. When a female anopheles mosquito ingests the blood of a host containing malaria parasites, the RBCs and asexual stage parasites are digested, while the gametocytes undergo further development to form macrogametocytes (female) or microgametocytes (male). In the mosquito gut, the male and female gametes fuse to form a diploid ookinete (the parasite is haploid during the rest of the lifecycle). As the oocyst matures, it divides to produce sporozoites, which travel to the salivary glands and are able to infect a new host when the mosquito next takes a blood meal.
Antimalarial drug development strategies often focus on targeting the asexual stages of Plasmodium development [6,11-13]. These intraerythrocytic stages are metabolically active and display a number of biochemical pathways that are unique to the parasite. Although most of the currently available antimalarial drugs target asexual malaria parasites, they have different activities, pharmacokinetic characteristics and toxicity profiles. For these reasons treatment recommendations vary depending on the nature of the disease. The WHO currently classifies malaria treatment into two categories: uncomplicated malaria and severe malaria [102]. To address these recommendations, organizations such as the Medicines for Malaria Venture have created seven drug target profiles [14]:

- Treatment of uncomplicated $P$. falciparum malaria

- Non-oral treatment of complicated/severe P. falciparum malaria in adults and children

- Treatment of P. vivax malaria

- Intermittent preventative treatment (IPT) in pregnant women

- IPT in infants 
- Stand-by treatment in travelers

- Chemoprophylaxis

Unfortunately, the discovery and development of new antimalarial drugs is a very long and involved process and in the end may not be successful for a number of reasons, including cost of synthesis, bioavailability and off-target effects. Many drugs aimed at the developed world are too expensive to be used in the low-cost environments that are characteristic of malaria endemic areas. However, a number of different strategies can be applied to antimalarial drug development to reduce cost. These include structural modification of existing drugs and 'piggyback' approaches that utilize drugs that have already been developed for other organisms or diseases, or act on a common target. An alternative is the de novo identification of drugs that act against a novel target, or a combination of these approaches. Focusing on parasite-specific targets for which no human host homologue exists reduces the chance of drug toxicity. In 2002, the complete sequence of the first $P$. falciparum clone was published [15]. This wealth of information has been instrumental in the identification of new molecular targets for the development of novel antimalarial chemotherapeutic agents. This review highlights the ongoing development of the targets that the authors have been investigating as potential avenues to exploit in the development of new drugs.

There are a number of new antimalarial agents in preclinical and clinical development that target both novel and classical parasite biochemical pathways (reviewed elsewhere [16-19]). Here, we discuss some different parasite molecular targets that may well lead to the future development of new chemotherapeutic agents.

\section{'Piggyback' drug discovery for malaria}

The piggyback antimalarial approach aims to identify agents that are either currently marketed or are being developed for treatment of other diseases, and usually have a common, or orthologous, target. An advantage of this approach is a potential cost-saving based on previous drug development investment for other diseases. However, given that drugs developed for diseases of wealthy nations are often expensive, even if they are relatively cheap to produce, some drugs may not be applicable to this approach unless patents have expired; for example, some antibiotics such as tetracyclines, which have antimalarial properties [20]. A good example of the piggyback approach in action is the exploitation of atovaquone. While this compound was initially shown to be an antimalarial, its activity against Pneumocystis led to it being re-explored as an antimalarial, and now atovaqoune combined with proguanil (Malarone ${ }^{\mathrm{TM}}$ ) is approved for malaria treatment and chemoprophylaxis [21]. Current examples of compounds being explored under the antimalarial piggyback approach are histone deacetylase (HDAC) inhibitors and HIV aspartic protease inhibitors (APIs).

\section{Histone deacetylase inhibitors: targeting gene transcription}

Histone deacetylases are enzymes that are involved in regulating eukaryotic cell chromatin structure, transcription and gene expression. They act together with histone acetlytransferases and other proteins to alter the acetylation of the lysine side chains of histones [22-24]. Paradoxically, the inhibition of histone deacetylation can both activate and suppress the transcription of genes [25,26]. HDAC inhibitors have been widely evaluated for their therapeutic properties (for recent reviews see [27,28]) and so they might represent attractive targets for antimalarial drugs that act by a novel mechanism.

While there are at least five HDAC homologues/orthologues in P. falciparum [29-34], the focus of drug discovery efforts to date has been on the PfHDAC1 protein. PfHDAC1 is expressed in intraerythrocytic-stage parasites [29] and appears to have some subtle differences in the predicted enzyme active site entrance compared with human enzymes, which are being exploited for antimalarial drug discovery [35-38]. Promising in vitro activities (low $\mathrm{nM} \mathrm{IC}_{50}$ values with very good selectivity in some cases) have recently been found for hydroxamate-class HDAC inhibitors against $P$. falciparum parasites [39]. Many of these compounds were originally designed for use as anticancer agents [40,41]. Mode-of-action studies show that this class of compounds displays all the hallmarks of HDAC inhibitors against malaria parasites, including hyperacetylation of parasite histones and inhibition of in situ and recombinant enzyme activities. Our ongoing development of this class of compounds is focusing on improving the pharmacokinetic profile.

\section{Taking advantage of the antimalarial activity of the HIV aspartic protease inhibitors}

It is becoming increasingly evident that the control of malaria depends not only on agents that target the intra-erythrocytic stages of parasite development, but also on the development of drugs that can inhibit additional stages of the parasite life cycle. Drugs that target gametocytes or at least prevent the development of these stages in the infected host are needed to control disease transmission, and drugs that target the pre-erythrocytic stages of parasite development are important as they can reduce the incidence of relapses and/or prevent infection altogether. Despite the growing appreciation of the need to target additional life cycle stages, most of the currently available antimalarial drugs and the majority of the drug development activities now underway focus on the asexual (blood) stages of parasite development $[16,17,19]$. One new group of antimalarial agents that might target multiple stages of Plasmodium development via a mechanism of action different to all of the currently available antimalarial drugs are HIV APIs.

A number of studies have demonstrated that some APIs inhibit malaria parasite growth [42] and that the antimalarial activity of these drugs occurs at clinically relevant concentrations [43]. APIs inhibit the growth of malaria parasites in mice [44] and sera taken from HIV patients receiving APIs inhibits the growth of $P$. falciparum in vitro [45]. Recent studies have demonstrated that APIs also possess activity against pre-erythrocytic stages [46]. Activity against a range of life cycle stages is unusual, and indicates that these drugs may represent a promising lead towards a new group of antimalarial agents that may reduce clinical disease and relapse. Their effect on gametocytogenesis and gametocytes has yet to be evaluated owing to the difficulty in evaluating drug studies on this stage of parasite development; although new methods are 
becoming available [47,48]. Very few antimalarial drugs are active against the pre-erythrocytic stage. The APIs saquinavir and ritonavir (at $4 \mu \mathrm{M}$ ) (FIGURE 2) also kill $P$. falciparum and $P$. vivax isolates taken directly from infected people [49]. These data, together with the in vivo data in mice [44], show that the antimalarial activity of APIs is not restricted to a single Plasmodium species.

In HIV, APIs inhibit the viral aspartyl protease, an essential enzyme for the production of mature HIV virions [50]. Data from a number of studies suggest that these drugs also kill malaria parasites by inhibiting an aspartyl protease (commonly referred to as a plasmepsin $[\mathrm{PM}]$ in malaria). The $P$. falciparum genome encodes ten aspartyl proteases. Four of these PMs are located in the food (digestive) vacuole (DV; PMI, PMII, PMIV and the histo-aspartic protease [HAP]) and are involved in hemoglobin digestion [51]. The six additional PMs have unknown functions. While recombinant enzyme assays demonstrate that high concentrations of the APIs saquinavir, lopinavir and ritonavir can inhibit the activity of the DV PMs PMII and -IV [52], other data support the hypothesis that these PMs are not the primary antimalarial targets of these drugs. Evidence for this hypothesis includes the demonstration that ritonavir and saquinavir behave antagonistically with E64, a well-characterized cysteine protease inhibitor, a characteristic that we would not expect of a DV PM inhibitor [43]. Furthermore, when the DV PMs PMI-IV are knocked out, both individually and collectively, the transgenic knockout parasites remain viable and retain their sensitivity to APIs [53-55].

Bootstrap analysis of the PMs suggests that the DV and non-DV PMs are not derived from a common ancestor in Apicomplexans [51], and may indicate a separate function for both groups. PMI, -II and -IV and HAP are clustered on chromosome 14, are expressed at high levels in blood-stage parasites and are localized to the DV where they participate in the digestion of hemoglobin [51]. The six remaining PMs are not clustered and appear to have different patterns of expression. PMV and -X have no introns and are likely to be related to PMI-IV. PlasmoDB [103] data suggest that $P$. falciparum PMs PMV, -VIII, -IX and -X are transcribed in blood-stage parasites. There was some discrepancy in the annotations of PMVIII by PlasmoDB and the Victorian Bioinformatics Consortium [104], with PlasmoDB predicting a much smaller protein; however, this now appears to have been rectified. While the expression profiles of PMVI and -VII have yet to be experimentally confirmed, PlasmoDB data indicate that these PMs are primarily expressed in gametocytes, and are thus likely to be of less interest as a target of an intra-erythrocytic drug. PMV, -IX and -X are the only non-DV PMs that have been investigated, and only a few studies exist [56,57]. These studies have shown that PMV, -IX and -X are present in blood-stage parasites [56]. PMV is believed to be an integral membrane protein located in the endoplasmic reticulum [57]. Searches of P. vivax and Plasmodium chabaudi sequence data show that genes encoding similar proteins to $P$. falciparum PMs PMV, -VI, -VII, -IX and -X exist in $P$. chabaudi and $P$. vivax [103].

Taken together, these data suggest that notwithstanding any inhibition of PMI, -II, -IV and HAP, the APIs exert a major role in killing malaria parasites by inhibiting one of the nonfood vacuole blood-stage aspartyl proteases that have essential biological functions external to the food vacuole. However, which of these nonvacuole PMs that is/are the target(s) of the APIs has yet to be experimentally verified. By identifying and characterizing the target of these drugs, we are paving the way for rational drug design. This process has been greatly aided by the numerous solid-state structures currently deposited in the Protein Data Bank database [105] and by the advances in in silico high-throughput screening [58], computational structure prediction methods [59], and molecular docking and scoring algorithms [60]. One problem that arises is that whist sequence identity of the Plasmodium aspartic proteases is relatively high across the family $(\sim 60 \%)$ [61], structural variation around the active site means that substrate specificity across the group is variable [62]. Classically, this would require the assembly of several different drug-like molecules - one for each target protein. Using solid state x-ray data and 3D-modeling techniques, Valiente et al. have reported the key seven residues (Y17, V105, T108, L191, L242, Q275 and T298 based on PMII numbering) required for PM function (Figure 3) [63].

These amino acids are conserved across the malarial strains but not in human aspartic proteases, suggesting that novel and selective antimalarial drugs can be developed that can better utilize these structural differences and may even allow the assembly of 'adaptive' drug molecules that have the ability to inhibit several members of a protein family at the same time [64]. This approach would not only reduce the ability of the organism to utilize redundant PM pathways for hydrolysis but, additionally, would drastically reduce the ability of the parasite to raise drug mutants as the organism would have to adapt multiple proteins concurrently. This has been realized to some extent in systems described by Nezami $e t$ al. who, using computer modeling and docking techniques, were able to rationalize the actual binding energies of a set of allophenylnorstatine-based compounds towards the four PM proteins outlined previously [65].

\section{Targeting de novo parasite pathways: purine salvage}

One approach to the development of new antimalarial drugs is to exploit differences in the basic biology of the host cell and the parasite for the de novo identification of drugs that act against a novel target. One such target is the purine salvage pathway of $P$. falciparum. In mammals, purine synthesis can occur de novo or by interconversion of salvaged purines, whereas the malaria parasite is totally dependent on purine salvage using purine bases obtained from the host. Like all intracellular parasites, $P$. falciparum is auxotrophic for purines and possesses elaborate purine salvage pathways to scavenge purines from their hosts [66]. A key role in this overall salvage process is played by the parasite's purine phosphoribosyltransferases (PRTases). These enzymes catalyze the synthesis of purine nucleoside monophosphates from 5-phospho- $\alpha$-D-ribsosyl-1pyrophosphate (Prib-PP) and the corresponding purine base [67-69] .

One PRTase enzyme that has specificity for a number of purine bases is hypoxanthine-xanthine-guanine phosphoribosyl transferase (HXGPRT), which can utilize hypoxanthine, guanine and xanthine as substrates ( $\mathrm{Km} \mathrm{0.46,0.30} \mathrm{and} 29 \mu \mathrm{M}$, respectively) [68]. HXGPRT catalyzes the synthesis of the 6-oxopurine mononucleotides IMP, XMP and GMP by transferring the nitrogen base 


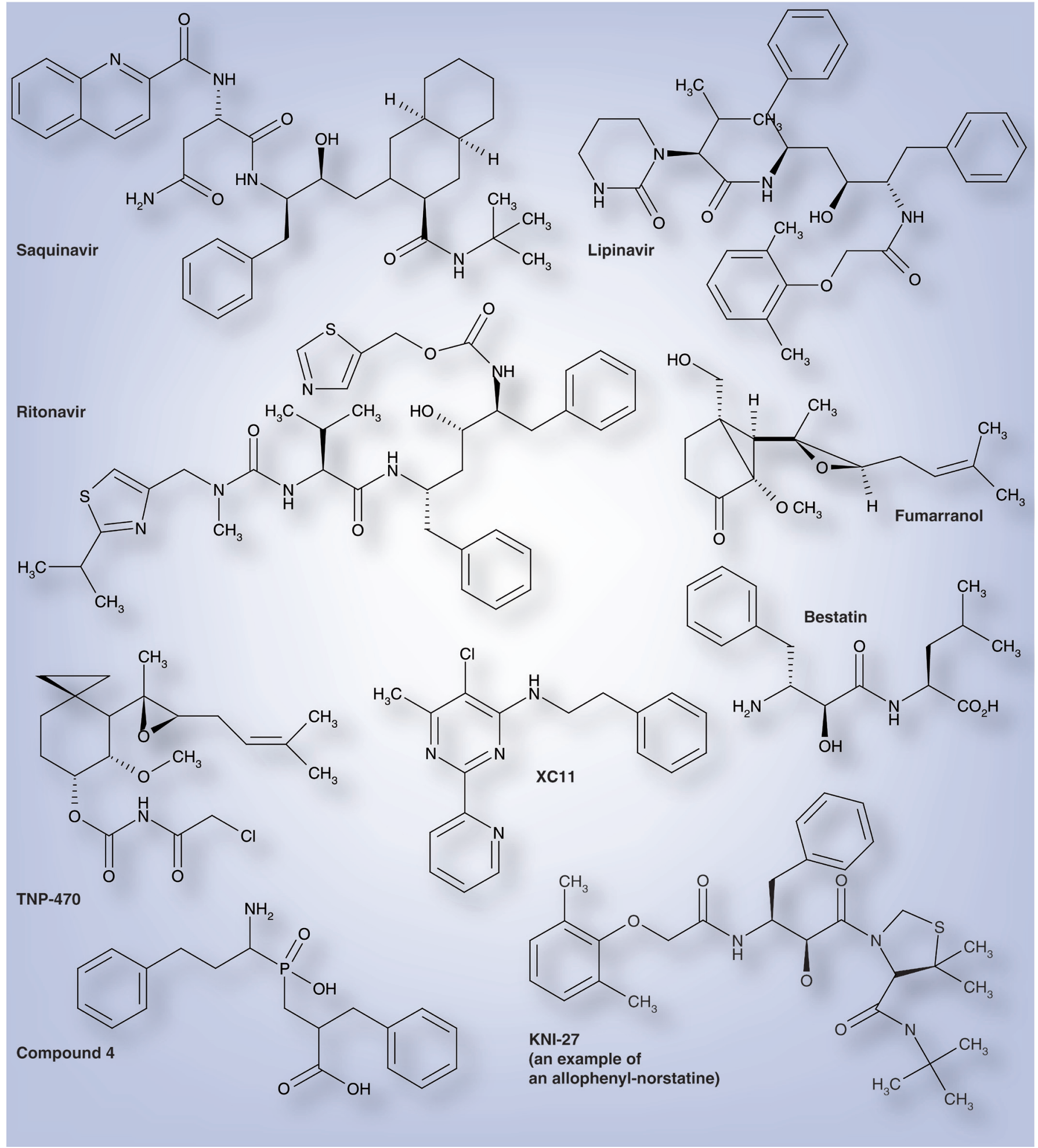

Figure 2. HIV aspartic protease inhibitors and aminopeptidase inhibitors.

to the $1-\beta$-position of the ribose ring of Prib-PP concomitant with the release of the pyrophosphate $\left(\mathrm{PP}_{\mathrm{i}}\right)$ moiety. The mechanism by which this is accomplished is ordered: first, Prib-PP complexes with $\mathrm{Mg}^{2+}$, followed by the binding of the purine base. $\mathrm{PP}_{\mathrm{i}}$ then dissociates from the complex and the nucleoside monophosphate is released [70]. Like other metallo-enzymes, for catalysis to occur a divalent metal ion is required, usually $\mathrm{Mg}^{2+}[67]$.

HGXPRTs have several common structural features, including a conserved core domain and a less conserved hood domain. The active site is located at the boundary of these two domains. It 


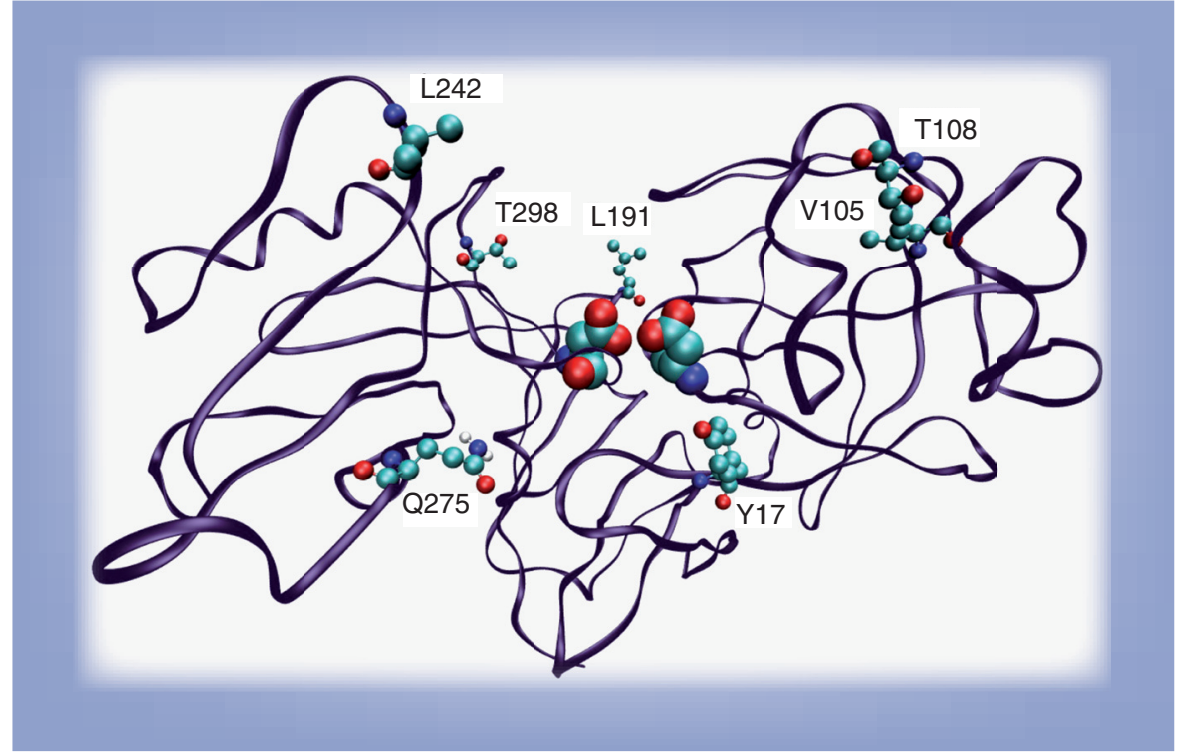

Figure 3. Ribbon representation of the plasmepsin II protein. The seven key residues required for activity in the malarial family (as reported by [64]) are shown as Corey, Pauling and Koltun surfaces. The two catalytic aspartic acid residues (D 34 and 214) are shown as van der Waals surfaces and are located in the center of the image. Adapted from Protein Data Bank identification number 1LEE [104]. on structural patterns and substrate specificity. These enzymes remove amino acids from the unblocked N-termini of peptides and proteins and, in addition to their role in general protein and peptide metabolism, have more specific functions, including activation/inactivation of biologically active peptides, removal of the $\mathrm{N}$-terminal methionine of newly synthesized proteins and trimming of antigens for presentation by MHC-1 [76].

Investigation of the malaria genome database PlasmoDB [104] revealed that the annotated genome of $P$. falciparum contains eight aminopeptidases. Four of these are methionine aminopeptidases. The remaining four include two neutral aminopeptidases, an aspartyl aminopeptidase and a prolyl aminopeptidase, all of which are potentially involved in regulating the pool of amino acids inside the malaria cell.

\section{Methionine aminopeptidases}

Methionine aminopeptidases (MetAP) are includes regions for the binding of the components of the reaction, including the phosphate from the ribose- 5 '-phosphate group, the purine base, pyrophosphate and $\mathrm{Mg}^{2+}[69,70]$. Both the human and $P$. falciparum HGXPRT proteins have been crystallized in the presence of transition state analogue inhibitors [71,72]. Both the human and $P$. falciparum structures show nearly identical interactions between the enzyme and the inhibitors [71,72], suggesting that designing selective inhibitors may not be feasible. However, Keough et al. have shown that it is possible to design inhibitors that have higher selectivity for the $P$. falciparum enzyme than its human homologue [73].

A series of novel HXGPRT inhibitors has recently been described. These inhibitors are based on a class of nucleoside analogues called acyclic nucleoside phosphonates [74] and are up to 60-times more selective for the $P$. falciparum HXGPRT than the human enzyme. Although the selectivity of these inhibitors may need to be improved, this achievement bodes well for the development of a new class of antimalarial agents that target $P$. falciparum HGXPRT. This is particularly true given the observation that while the $P$. falciparum HXGPRT may be essential for the parasite, this is not the case in the human host where purine synthesis can occur either by de novo synthesis or via purine salvage. Thus, even if novel HXGPRT inhibitors do target the human host, they are unlikely to cause significant toxicity, as human patients with significantly reduced enzymatic function show few ill effects [75].

\section{Targeting aminopeptidases important to protein anabolism \& catabolism}

Aminopeptidases are exopeptidases that are distributed widely in prokaryotes and eukaryotes as either integral membrane or cytosolic proteins, and are classified into different classes based metalloproteases that are highly conserved in all organisms. Their function is to catalytically remove $\mathrm{N}$-terminal initiator methionines during protein synthesis, a process that is essential for the correct folding and trafficking of proteins. Two types of MetAP exist, type one (MetAP1) and type 2 (MetAP2), which are distinguished by the presence of a 60-amino acid insertion near the catalytic domain of MetAP2. There may be some functional redundancy between these two types of enzymes (e.g., in yeast) as they exhibit tissue- or cell-specific expression in multicellular organisms. Nonetheless, blocking the activity of both enzyme classes (separately or together) can be lethal to cell proliferation, and inhibitors such as TNP-470 have been considered as potential anticancer compounds [77].

Malaria parasites express four MetAP enzymes, three MetAP1 isoforms (designated PfMetAP1a, b and c) and one MetAP2 isoform. Zhang et al. first reported that the MetAP inhibitor fumagillin and its derivative TNP-470 potently blocked the growth of $P$. falciparum malaria (and Leishmania donovani) parasites in culture and surmised that the target for killing was PfMetAP2, which they isolated and cloned [78]. More recently, Chen et al. demonstrated that a novel derivative fumarranol, which exhibits far less toxicity in mice than TNP-470 (no skin lesions), not only killed $P$. falciparum malaria parasites in culture but also inhibited the growth of Plasmodium yoelii (17X lethal strain) in mice. Fumarranol binds to recombinant PfMetAP2 but not to human MetAP2 in a noncovalent reversible manner. Furthermore, neither fumarranol nor TNP-470 bound to PfMetAP1a, b and c, demonstrating their selectivity for PfMetAP2 [77].

Chen and colleagues also expressed the three PfMetAP1s of $P$. falciparum as functionally active recombinant proteins, and then employed PfMetAP1b, because of its close sequence identity to the 
human and yeast MetAP1, in a high-throughput drug-screening program [79]. From this they identified a previously uncharacterized structural class of inhibitors containing a pyridinyl-pyrimidine core. The most potent compound, XC11, demonstrated a tenfold greater activity against PfMetAP1b $\left(\mathrm{IC}_{50}: 0.7 \mu \mathrm{M}\right)$ than against human MetAP2 $\left(\mathrm{IC}_{50}: 7.0 \mu \mathrm{M}\right)$, and exhibited little toxicity against human fibroblasts in a cell proliferation assay $\left(\mathrm{IC}_{50}: 75 \mu \mathrm{M}\right)$. XC11 was also over 100-fold more selective for the inhibition of PfMetAP1b when compared with PfMetAP1a and c. Most importantly, XC11 was active against both chloroquine-susceptible and -resistant P. falciparum in culture and Plasmodium berghei $\mathrm{K} 173$ in mice [79].

It follows that inhibitors against MetAP1 and MetAP2 of malaria parasites have potential as new antimalaria drugs, with the added prospect of creating potent crossover or combined therapies that target several isoforms. Inhibitors of these enzymes could also be combined with inhibitors of other aminopeptidases (see later), in the event that this provides added benefit in either synergistic antimalarial activity or protection against the development of drug-resistant parasites.

\section{Neutral aminopeptidases}

Two neutral aminopeptidases have been identified in the annotated $P$. falciparum genome, a M1-family alanyl aminopeptidase (PfM1AAP) and a M17-family leucine aminopeptidase (PfM17LAP).

Functionally active recombinant PfM1AAP and PfM17LAP have been produced and characterized [80,81]. Biochemical studies of parasite extracts and the recombinant proteins indicate that these two enzymes can account for all of the neutral aminopeptidase activity characterized in soluble $P$. falciparum cytosolic extracts [80,81]. Assays using peptide substrates have revealed that while PfM1AAP and PfM17LAP are members of different protease families, they have overlapping substrate specificities. However, PfM1AAP exhibits broader substrate specificity than PfM17LAP. PfM1AAP's broad substrate specificity is consistent with other M1 aminopeptidases, including the aminopeptidase Ns from Grampositive bacteria [82]. In contrast to PfM1AAP, PfM17LAP preferentially cleaves substrates with $\mathrm{N}$-terminally exposed hydrophobic amino acids, particularly leucine [80], While a preference for leucine substrates has been described as a general feature of M17 leucyl aminopeptidases, PfM17LAP has a narrower substrate specificity than other characterized multimeric eukaryotic LAPs [83]. The observation that PfM1AAP and PfM17LAP are redundant in their ability to release leucine from peptides suggests that this is a critical function within the malaria parasite. One possibility is that the leucine obtained by digestion of host hemoglobin-derived peptides (or other proteins) may be exchanged for extracellular isoleucine [84], the only amino acid not present in human hemoglobin, and one that cannot be synthesized by the parasite $[85,86]$.

Bestatin, a fungal-derived antibiotic and a natural analog of the dipeptide Phe-Leu, is a potent inhibitor of $P$. falciparum neutral aminopeptidase activity. Bestatin inhibits the growth of $P$. falciparum both in vitro and in vivo [87,88], and appears to inhibit both PfM1AAP and PfM17LAP by chelating the active metal ions within their metal-binding centres $[81,89,90]$. Rationally designed phosphinate dipeptide analogs [81] also inhibit recombinant forms of these enzymes (rPfM1AAP and rPfM17LAP) more effectively than bestatin and are also active against $P$. falciparum in culture [89]. Furthermore, in vivo studies using a nonlethal Plasmodium chabaudi chabaudi murine malaria model have demonstrated that at least one of these compounds can reduce the parasite burden by up to $92 \%$, as well as produce a 3 -day delay in the appearance of parasites [89]. However, it is not clear which of the two enzymes plays a more significant role in parasite survival.

Transgenic parasites that overexpress each of the $P$. falciparum neutral aminopeptidases have been generated in an attempt to determine which, or if both, of these enzymes are the antimalarial target of bestatin and the phosphinate dipeptide analogs [81,91]. These studies have demonstrated that increased expression of either enzyme can protect them from drug-induced growth inhibition by these drugs and suggest that both $P$. falciparum neutral aminopeptidases are targeted by these drugs. The data also highlight the need to investigate the similarities and differences in the active site of these enzymes to enhance the prospects of developing potent antimalarial agents. The overlapping specificities of PfM1AAP and PfM17LAP for substrates could be exploited in the design of either specific and/or dual-acting inhibitors. Two specific inhibitors used in combination, or ideally a dual inhibitor, could have increased potency. Both enzymes are encoded by single-copy genes and no other neutral aminopeptidases exist in malaria parasites, indicating that issues of functional redundancy experienced with the DV proteases are unlikely to be a problem.

The x-ray crystal structure of rPfM1AAP was recently published [81] and has revealed some important characteristics that may allow the development of potent inhibitors of this enzyme. The zinc-binding and substrate-coordinating motifs of the enzyme are buried within catalytic domain II and have access to the external environment via two openings. The first opening is formed by domain IV, a helical and the C-terminal portion of the protease, and is large enough to permit substrate entry to the active site. The second opening formed by domains I and IV is much smaller and may represent the exit portal for released amino acids. The structure of rPfM1AAP also suggests that, in addition to the rational design of active site inhibitors, the creation of agents that block active site access (entry and exit) may be feasible. For example, blocking the exit of the released amino acids from this enzyme may prevent substrate access to the buried active site.

\section{Aspartyl aminopeptidase (M18AAP) \& prolyl aminopeptidase (S33PAP)}

The two remaining aminopeptidases identified within the $P$. falciparum genome are an aspartyl and prolyl aminopeptidase. Compared with the two neutral aminopeptidases, M18AAP and S33PAP have a highly restricted substrate preference. M18AAP cleaves aspartic acid and, less so, glutamic acid from the $\mathrm{N}$-terminus of proteins, while S33PAP will remove proline residues only [92].

The aspartyl aminopeptidases are classified as belonging to the M18 class. Unlike the M1 and M17 neutral aminopeptidases [80,81], few M18 class aspartyl aminopeptidases have been characterized. Our relatively poor understanding of these enzymes stems from the general lack of available substrate and inhibitor reagents; at present, 
there is only limited information reported for the aspartyl amino peptidase of mammals [93], yeast [94] and bacteria [106].

The restricted specificity of M18AAP for the N-terminal acidic amino acids, aspartic and glutamic acid, which cannot be cleaved by any other aminopeptidases, makes them of particular interest. Owing to this specificity, it is assumed that they are involved in a housekeeping function in protein turnover, acting in concert with the cytosolic neutral aminopeptidases. We have proposed that the P. falciparum M18AAP works alongside the malarial M1 and M17 neutral aminopeptidases in the terminal stage of hemoglobin digestion, releasing free amino acids that are used by the rapidly growing intra-erythrocytic parasites for protein anabolism $[80,90,91,95]$. While the neutral aminopeptidases can release most hydrophobic and hydrophilic amino acids, they are ineffective against acid amino acids and, therefore, require the assistance of M18AAP.

However, a recent report indicates that $P$. falciparum M18AAP is not only expressed in the cytosol but is also exported to the parasitophorous vacuole of the parasite. This suggests that M18AAP may play an additional role, possibly in aiding rupture of the parasite from the cell. In support of this idea, Lauterbach et al. [96] , using phage-display libraries, showed that the M18AAP bound to the human erythrocyte spectrin (a cytoskeletal) protein, and have subsequently identified a putative spectrin-binding motif [94]. Inhibition of the $P$. falciparum M18AAP using antisense RNA results in parasite growth abnormalities, vacuolarization and cellular damage [95]. Clearly, the M18AAP represents a target at which novel antimalarials that block its function(s) could be designed.

Similar to M18AAP, S33PAP has a highly restricted substrate preference. S33PAP can only remove proline residues from the $\mathrm{N}$-terminus of proteins. Microarray analysis indicates that the expression of S33PAP is switched on earlier in the parasites development within the RBC than the other three non-methionine aminopeptidases and, therefore, this enzyme may have additional or separate function(s) to these enzymes. S33PAP is of particular interest as a potential target for drug development since, although detected in many microbes, this activity and genes encoding S33PAP enzymes have not been detected in mammals. Accordingly, S33PAP may be particularly attractive for the design of antimalarial inhibitors. While very little is known about the biology or biochemical characteristics of this enzyme, gene sequence analysis of the conceptually translated protein has revealed the presence of a protein export element (PEXEL) or vacuolar transit sequence thought to be involved in the transport of proteins across the parasitophorus vacuole membrane and into the red cell cytosol [97,98].

\section{Conclusion}

Malaria remains one of the most lethal diseases to humans. A new momentum for a global eradication program is gaining pace. However, drug resistance by the parasite is an ongoing issue, and without the advent of new and novel chemotherapeutic agents, this current eradication program may end in the same fashion as the last eradication program of the 1950 s, with some successes but also many failures. The genome of $P$. falciparum has been sequenced and a number of potential drug targets have been identified. Rational drug design using both piggyback techniques as well as de novo identification will both play a role in the design of new antimalarial agents. Whether any of these agents will eventually become firstline drugs against the parasite depends on a number of factors, such as cost of raw materials, ease of synthesis, pharmacokinetics, stability and toxicity. Nonetheless, combination therapy to prevent the emergence of parasite resistance is also widely accepted as the only prudent course, and some of the targets outlined in this review may become part of combination therapies with existing agents.

\section{Expert commentary}

Given the urgent need to develop new antimalaria drugs, it is imperative that researchers identify novel biochemical pathways in malaria parasites that when disrupted with compounds are lethal to the parasite. The availability of the malaria genome (PlasmoDB) offers an invaluable platform from which these can be identified and from which molecular targets can be selected. However, production of functionally active recombinant forms of potential targets that can be exploited in structure analysis, the development of robust molecular assays and high-throughput drug screening present a major but not insurmountable problem. In this review, we have highlighted several examples of such targets, which may lead to the development of new antimalarial drugs, although there is a long way to go to before we inhibitory compounds that can be synthesized cheaply and have no off-target effects. Nevertheless, the continued investigation of such molecular targets adds to our database on what is and is not worth pursuing.

\section{Five-year view}

Malaria remains a devastating disease, with drug resistance an ongoing concern, particularly with reports of increased tolerance to artemisinin in southeast Asia. There is a renewed emphasis on global 'eradication', and while this may be unrealistic in the short term, there has been significant progress in the last few years towards reducing the global burden of disease. There are currently a number of new chemotherapeutic agents/targets in the drug 'pipeline' at different stages of development. Some, like those outlined in this review, are still in the discovery phase and, realistically, a number of targets and their inhibitors will not progress further for reasons that plague all drug discovery programs. Nonetheless, within 5 years, the potential for a number of new or novel antimalarial agents to have been validated for use in humans is high. As the identification of novel proteins and metabolic processes unique to the parasite increases, new potential targets will also become available for investigation, thus preventing the same type of failures that beset the first global eradication program when chloroquine resistance first emerged.

\section{Financial \& competing interests disclosure}

The authors thank the National Health and Medical Research Council of Australia, the Australian Research Council and Griffith University for funding support. The authors have no other relevant affiliations or financial involvement with any organization or entity with a financial interest in or financial conflict with the subject matter or materials discussed in the manuscript apart from those disclosed.

No writing assistance was utilized in the production of this manuscript. 


\section{Key issues}

- Malaria parasites infect millions of people in the tropics and subtropics, and every year approximately 1 million of these infections result in death.

- The prevention and treatment of malaria is becoming more difficult due to the global spread of drug-resistant parasites, and new antimalarial agents that act against novel parasite targets are needed.

- A number of strategies can be applied to antimalarial drug development. The 'piggyback' approach uses drugs already developed for other organisms or diseases and act on a common target. The de novo approach exploits differences in the basic biology of the host cell and the parasite in order to identify new drugs that act on a novel target. These approaches can be used in combination.

- Current examples of compounds being explored under the piggyback approach include histone deacetylase (HDAC) inhibitors and HIV aspartic protease inhibitors.

- HDAC inhibitors were originally designed for use as anticancer agents and are thought to act against malaria parasites by hyperacetylation of parasite histones and inhibition of enzyme activity. HIV aspartic protease inhibitors are currently used to treat HIV infection and are thought to kill malaria parasites by inhibition of an as yet unidentified essential nonfood vacuole, blood-stage aspartyl protease.

- Current examples of targets being explored under the de novo approach include the purine salvage pathway enzyme hypoxanthinexanthine-guanine phosphoribosyltransferase and aminopeptidases important to protein anabolism and catabolism

- Several aminopeptidases are also promising targets for the development of novel antimalarial drugs. These include the methionine aminopeptidases (PfMetAP1 and PfMetAP2), the neutral aminopeptidases (alanyl and leucyl), and aspartyl and prolyl aminopeptidases.

- Compounds that selectively block malaria methionine aminopeptidase 1b (PfMetAP1b) activity and exhibit activity against rodent malaria models in vivo have been identified. Similarly, compounds that target the neutral aminopeptidases in vitro and exhibit activity against rodent malaria models in vivo have also been identified.

\section{References}

Papers of special note have been highlighted as:

- of interest

-• of considerable interest

1 Enserink M. Epidemiology. Lower malaria numbers reflect better estimates and a glimmer of hope. Science 321(5896), 1620 (2008).

2 Dev V, Phookan S, Sharma VP, Dash AP, Anand SP. Malaria parasite burden and treatment seeking behavior in ethnic communities of Assam, northeastern India. J. Infect. 52(2), 131-139 (2006).

3 Nosten F, Rogerson SJ, Beeson JG, McGready R, Mutabingwa TK, Brabin B. Malaria in pregnancy and the endemicity spectrum: what can we learn?

Trends Parasitol. 20(9), 425-432 (2004).

4 Galinski MR, Barnwell JW. Plasmodium vivax: who cares? Malar. J. 7(Suppl. 1), S9 (2008).

5 Jefford CW. Why artemisinin and certain synthetic peroxides are potent antimalarials. Implications for the mode of action. Curr. Med. Chem. 8, 1803-1826 (2001).

6 Gardiner DL, McCarthy JS, Trenholme KR. Malaria in the postgenomics era: light at the end of the tunnel or just another train. Postgrad. Med. J. 81, 505-509 (2005)

7 Aweeka FT, German PI. Clinical pharmacology of artemisinin-based combination therapies. Clin.

Pharmacokinet. 2, 91-102 (2008).
8 Uhlemann AC, Krishna S. Antimalarial multi-drug resistance in Asia: mechanisms and assessment. Curr. Top. Microbiol. Immunol. 295, 39-53 (2005)

9 Mugittu K, Ndejembi M, Malisa A et al. Therapeutic efficacy of sulfadoxinepyrimethamine and prevalence of resistance markers in Tanzania prior to revision of malaria treatment policy: Plasmodium falciparum dihydrofolate reductase and dihydropteroate synthase mutations in monitoring in vivo resistance. Am. J. Trop. Med. Hyg. 71, 696-702 (2004)

10 Marks F, von Kalckreuth V, Kobbe R et al. Parasitological rebound effect and emergence of pyrimethamine resistance in Plasmodium falciparum after single-dose sulfadoxine-pyrimethamine. J. Infect. Dis. 192, 1962-1965 (2005).

11 Rogers WO, Sem R, Tero T et al. Failure of artesunate-mefloquine combination therapy for uncomplicated Plasmodium falciparum malaria in southern Cambodia. Malar. J. 8, 10 (2009).

12 Wongsrichanalai C, Meshnick SR. Declining artesunate-mefloquine efficacy against falciparum malaria on the Cambodia-Thailand border. Emerg. Infect. Dis. 14, 716-719 (2008).

- Indicates that resistance to artesunate may soon be a significant problem in southeast Asia.

13 Schlitzer M: Malaria chemotherapeutics part I: history of antimalarial drug development, currently used therapeutics, and drugs in clinical development. ChemMedChem 2(7), 944-986 (2007).
14 Bathurst I, Hentschel C. Medicines for malaria venture: sustaining antimalarial drug development. Trends Parasitol. 22, 301-307 (2006).

15 Gardner MJ, Hall N, Fung E et al. Genome sequence of the human malaria parasite Plasmodium falciparum. Nature 419, 498-511 (2002).

16 Fidock DA, Eastman RT, Ward SA, Meshnick SR. Recent highlights in antimalarial drug resistance and chemotherapy research. Trends Parasitol. 24, 537-544 (2008).

- Overview of some current drug research highlighted at the Molecular Approaches to Malaria Meeting in Australia.

17 Jana S, Paliwal J. Novel molecular targets for antimalarial chemotherapy. Int. J. Antimicrob. Agents 30, 4-10 (2007).

18 Rosenthal PJ. Antimalarial drug discovery: old and new approaches. J. Exp. Biol. 206, 3735-3744 (2003).

- Good overview of malarial drug discovery.

19 Schlitzer M. Antimalarial drugs - what is in use and what is in the pipeline. Arch. Pharm. (Weinheim) 341, 149-163 (2008).

20 Clough B, Wilson RJM. Antibiotics and the plasmodial plastid organelle. In: Antimalarial Chemotherapy: Mechanisms of Action, Resistance, and New Directions in Drug Discovery. Rosenthal PJ (Ed.). Humana Press, NJ, USA, 265-286 (2001).

21 Canfield CJ, Pudney M, Gutteridge WE. Interactions of atovaquone with other antimalarial drugs against Plasmodium falciparum in vitro. Exp. Parasitol. 80(3), 373-381 (1995). 
22 Phillips DM. The presence of acetyl groups of histones. Biochem. J. 87, 258-263

(1963).

23 Allfrey VG, Faulkner R, Mirsky AE. Acetylation and methylation of histones and their possible role in the regulation of RNA synthesis. Proc. Natl Acad. Sci. USA 51, 786-794 (1964).

24 Gershey EL, Vidali G, Allfrey VG. Chemical studies of histone acetylation. The occurrence of epsilon- $N$-acetyllysine in the f2a1 histone. J. Biol. Chem. 243, 5018-5022 (1968).

25 Van Lint C, Emiliani S, Verdin E. The expression of a small fraction of cellular genes is changed in response to histone hyperacetylation. Gene Expr. 5, 245-253 (1996).

26 Mitsiades CS, Mitsiades NS, McMullan CJ et al. Transcriptional signature of histone deacetylase inhibition in multiple myeloma: biological and clinical implications. Proc. Natl Acad. Sci. USA 101, 540-545 (2004).

27 Gallinari P, Di Marco S, Jones P, Pallaoro M, Steinkuhler C. HDACs, histone deacetylation and gene transcription: from molecular biology to cancer therapeutics. Cell Res. 17, 195-211 (2007).

28 Rasheed WK, Johnstone RW, Prince HM. Histone deacetylase inhibitors in cancer therapy. Expert Opin. Investig. Drugs 16, 659-678 (2007).

29 Joshi MB, Lin DT, Chiang PH et al. Molecular cloning and nuclear localization of a histone deacetylase homologue in Plasmodium falciparum. Mol. Biochem. Parasitol. 99, 11-19 (1999).

30 Freitas-Junior LH, Hernandez-Rivas R, Ralph SA et al. Telomeric heterochromatin propagation and histone acetylation control mutually exclusive expression of antigenic variation genes in malaria parasites. Cell 121, 25-36 (2005).

31 Duraisingh MT, Voss TS, Marty AJ et al. Heterochromatin silencing and locus repositioning linked to regulation of virulence genes in Plasmodium falciparum. Cell 121, 13-24 (2005).

32 Merrick CJ, Duraisingh MT. Plasmodium falciparum Sir2: an unusual sirtuin with dual histone deacetylase and ADPribosyltransferase activity. Eukaryot. Cell 6, 2081-2091 (2007).

33 Fan Q, An L, Cui LP. fADA2, a Plasmodium falciparum homologue of the transcriptional coactivator ADA2 and its in vivo association with the histone acetyltransferase PfGCN5. Gene 336, 251-261 (2004).

34 Fan Q, An L, Cui L. Plasmodium falciparum histone acetyltransferase, a yeast GCN5 homologue involved in chromatin remodeling. Eukaryot. Cell 3, 264-276 (2004).

35 Andrews KT, Tran TN, Lucke A et al. Potent antimalarial activity of histone deacetylase inhibitor analogues. Antimicrob. Agents Chemother. 52, 1454-1461 (2008).

36 Cui L, Miao J, Furuya T, Li X, Su XZ, Cui L. PfGCN5-mediated histone H3 acetylation plays a key role in gene expression in Plasmodium falciparum. Eukaryot. Cell 6, 1219-1227 (2007).

37 Bozdech Z, Chaal B. Global transcriptional responses of Plasmodium falciparum to small molecular inhibitors. Abstracts of the Molecular Approaches to Malaria Conference. February 3-7, 2008. Lorne, Victoria, Australia. Int. J. Parasitol. 38(Suppl. 1), S53-S54 (2008).

38 Mukherjee P, Pradhan A, Shah F, Tekwani BL, Avery MA. Structural insights into the Plasmodium falciparum histone deacetylase 1 (PfHDAC-1): a novel target for the development of antimalarial therapy. Bioorg. Med. Chem. 16, 5254 5265 (2008).

39 Dow GS, Chen Y, Andrews KT et al. Antimalarial activity of phenylthiazolylbearing hydroxamate-based histone deacetylase inhibitors. Antimicrob. Agents Chemother. 52(10), 3467-3477 (2008).

40 Gallinari, P, Di Marco S, Jones P, Pallaoro M, Steinkuhler C. HDACs, histone deacetylation and gene transcription: from molecular biology to cancer therapeutics. Cell Res. 17, 195-211 (2007).

41 O'Connor OA, Heaney ML, Schwartz L et al. Clinical experience with intravenous and oral formulations of the novel histone deacetylase inhibitor suberoylanilide hydroxamic acid in patients with advanced hematologic malignancies. J. Clin. Oncol. 24, 166-173 (2006).

42 Skinner-Adams TS, McCarthy JS, Gardiner DL, Hilton PM, Andrews KT. Antiretrovirals as antimalarials. J. Infect. Dis. 190, 1998-2000 (2004).

- First publication identifying the antimalarial activity of HIV protease inhibitors.

43 Skinner-Adams TS, Andrews KT, Melville L, McCarthy JS. Gardiner DL. Synergistic interactions of antiretroviral protease inhibitors saquinavir and ritonavir with chloroquine and mefloquine against Plasmodium falciparum in vitro. Antimicrob. Agents Chemother. 51(2), 759-762 (2007).

44 Andrews KT, Fairlie DP, Madala PK et al. Potencies of human immunodeficiency virus protease inhibitors in vitro against Plasmodium falciparum and in vivo against murine malaria. Antimicrob. Agents Chemother. 50, 639-648 (2006).

45 Redmond AM, Skinner-Adams TS, Andrews KT et al. Antimalarial activity of sera from subjects taking HIV protease inhibitors. AIDS 21, 763-765 (2007).

46 Hobbs CV, Voza T, Coppi A et al. HIV protease inhibitors inhibit the development of preerythrocytic-stage plasmodium parasites. J. Infect. Dis. 199(1), 134-141 (2009).

47 Dixon MWA, Peatey CL, Gardiner DL, Trenholme KR. A green fluorescent protein based assay for determining commitment to gametocytogenesis in Plasmodium falciparum. Mol. Biol. Parasitol. 163, 123-126 (2009).

48 Peatey CL, Skinner-Adams TS, Dixon MW, McCarthy JS, Gardiner DL, Trenholme KR. The effect of antimalarial drugs on Plasmodium falciparum gametocytes. J. Infect. Dis. (2009) (In press).

49 Lek-Uthai U, Suwanarusk R, Ruengweerayut $\mathrm{R}$ et al. Stronger activity of human immunodeficiency virus type 1 protease inhibitors against clinical isolates of Plasmodium vivax than against those of $P$. falciparum. Antimicrob. Agents Chemother. 52(7), 2435-2441 (2008).

50 Tomasselli AG, Heinrikson RL. Targeting the HIV-protease in AIDS therapy: a current clinical perspective. Biochim. Biophys. Acta 1477(1-2), 189-214 (2000).

51 Coombs GH, Goldberg DE, Klemba M, Berry C, Kay J, Mottram JC. Aspartic proteases of Plasmodium falciparum and other parasitic protozoa as drug targets. Trends Parasitol. 17(11), 532-537 (2001).

52 Skinner-Adams TS, Guddat LW, Gardiner DL, McCarthy JS, Andrews KT. Are Plasmodium falciparum plasmepsins the target of HIV proteases? J. Infect. Dis. 191(8), 1382-1383 (2005).

53 Liu J, Gluzman IY, Drew ME, Goldberg DE. The role of Plasmodium falciparum food vacuole plasmepsins. J. Biol. Chem. 280(2), 1432-1437 (2005). 
54 Omara-Opyene AL, Moura PA, Sulsona CR et al. Genetic disruption of the Plasmodium falciparum digestive vacuole plasmepsins demonstrates their functional redundancy. J. Biol. Chem. 279(52), 54088-54096 (2004).

55 Bonilla JA, Moura PA, Bonilla TD, Yowell CA, Fidock DA, Dame JB. Effects on growth, hemoglobin metabolism and paralogous gene expression resulting from disruption of genes encoding the digestive vacuole plasmepsins of Plasmodium falciparum. Int. J. Parasitol. 37(3-4), 317-327 (2007).

56 Banerjee R, Liu J, Beatty W, Pelosof L, Klemba M, Goldberg DE. Four plasmepsins are active in the Plasmodium falciparum food vacuole, including a protease with an active-site histidine. Proc. Natl Acad. Sci. USA 99(2), 990-995 (2002).

57 Klemba M, Goldberg DE. Characterization of plasmepsin $\mathrm{V}$ a membrane-bound aspartic protease homolog in the endoplasmic reticulum of Plasmodium falciparum. Mol. Biochem. Parasitol. 143(2), 183-191 (2005).

58 Klebe G. Virtual ligand screening: strategies, perspectives and limitations. Drug Discov. Today 11 (13-14), 580-594 (2006).

59 Cavasotto CN, Phatak SS. Homology modelling in drug discovery: current trends and applications. Drug Discov. Today 14(13-14), 676-683 (2009).

60 Kitchen D, Decornez H, Furr J, Bajorath J. Docking and scoring in virtual screening for drug discovery: methods and applications. Nat. Rev. Drug Discovery 3(11) 935-949 (2004).

61 Asojo OA, Gulnik SV, Afonina E et al. Novel uncomplexed and complexed structures of plasmepsin II, an aspartic protease from Plasmodium falciparum. J. Mol. Biol. 327 (1) 173-181 (2003).

62 Beyer BB, Johnson JV, Chung AY et al. Active-site specificity of digestive aspartic peptidases from the four species of Plasmodium that infect humans using chromogenic combinatorial peptide libraries. Biochemistry 44 (6) 1768-1779 (2005).

63 Valiente PA, Batista PR, Pupo A, Pons T, Valencia A, Pascutti PG. Predicting functional residues in Plasmodium falciparum plasmepsins by combining sequence and structural analysis with molecular dynamics simulations. Proteins 73 (2) 440-457 (2008).
64 Freire E. Designing drugs against heterogeneous targets. Nat. Biotechnol. 20 (1), 15-16 (2002).

65 Nezami A, Kimura T, Hidaka K et al. High-affinity inhibition of a family of Plasmodium falciparum proteases by a designed adaptive inhibitor. Biochemistry 42 (28), 8459-8464 (2003).

66 Queen SA, Vander Jagt D, Reyes P. Properties and substrate specificity of a purine phosphoribosyltransferase from the human malaria parasite, Plasmodium falciparum. Mol. Biochem. Parasitol. 30, 123-134 (1988).

67 Keough DT, Ng AL, Winzor DJ, Emmerson BT, de Jersey J. Purification and characterization of Plasmodium falciparum hypoxanthine-guanine-xanthine phosphoribosyltransferase and comparison with the human enzyme. Mol. Biochem. Parasitol. 98, 29-41 (1999).

68 Gero AM, O'Sullivan WJ. Purine and pyrimidines in malaria parasites. Blood Cells 16, 467-484 (1990).

69 Sherman IW. Biochemistry of Plasmodium (malarial parasites). Micro. Reviews 43, 453-495 (1979).

70 Xu Y, Eads J, Sacchettini JC, Grubmeyer C. Kinetic mechanism of human hypoxanthineguanine phosphoribosyltransferase. Biochemistry 36, 3700-3712 (1997).

71 Shi W, Li CM, Tyler PC et al. The $2.0 \AA$ structure of human hypoxanthine-guanine phosphoribosyltransferase with a transition-state analog inhibitor. Nat. Struct. Biol. 6, 588-593 (1999).

72 Shi W, Li CM, Tyler PC et al. The $2.0 \AA$ structure of malarial purine phosphoribosyltransferase in complex with transition-state analogue inhibitor. Biochemistry 38, 9872-98780 (1999).

73 Keough DT, Skinner-Adams T, Jones MK et al. Lead compounds for antimalarial chemotherapy: purine base analogs discriminate between human and P. falciparum 6-oxopurine phosphoribosyltransferases. J. Med. Chem. 49, 7479-7486 (2006).

74 Keough DT, Hocková D, Holý A et al. Inhibition of hypoxanthine-guanine phosphoribosyltransferase by acyclic nucleoside phosphonates: a new class of antimalarial therapeutics. J. Med. Chem. 52(14), 4391-4399 (2009).

75 Dawson PA, Gordon RB, Keough DT, Emmersonc BT. Normal HPRT coding region in a male with gout due to HPRT deficiency. Mol. Genet. Metab. 85, 78-80 (2005).
76 Harris CA, Hunte B, Krauss MR, Taylor A, Epstein LB. Induction of leucineaminopeptidase by interferon- $\gamma$. Identification by protein microsequencing after purification by preparative twodimensional gel electrophoresis. J. Biol. Chem. 267, 6865-6869 (1992)

77 Chen X, Xie S, Bhat S, Kumar N, Shapiro TA, Liu JO. Fumagillin and famarranol interact with $P$. falciparum methionine aminopeptidase 2 and inhibit malaria parasite parasite growth in vitro and in vivo. Chem Biol. 16, 193-202 (2009).

78 Zhang P, Nicholson DE, Bujnicki JM et al. Angiogenesis inhibitors specific for methionine aminopeptidase 2 as drugs for malaria and leishmaniasis. J. Biomed. Sci. 9, 34-40 (2002).

79 Chen X, Chong CR, Lirong S, Yoshimoto T, Sullivan DJ Jr, Liu JO. Inhibitors of Plasmodium falciparum methionine aminopeptidase $1 \mathrm{~b}$ possess antimalarial activity. Proc. Natl Acad. Sci. USA 103, 14548-14553 (2006).

80 Stack CM, Lowther J, Cunningham E et al. Characterization of the Plasmodium falciparum M17 leucyl aminopeptidase. A protease involved in amino acid regulation with potential for antimalarial drug development. J. Biol. Chem. 282(3), 2069-2080 (2007).

81 McGowan S, Porter CJ, Lowther J et al. Structural basis for the inhibition of the essential Plasmodium falciparum M1 neutral aminopeptidase. Proc. Natl Acad. Sci. USA 106, 2537-2542 (2009).

- Crystal structure of the Plasmodium falciparum M1 aminopeptidase.

82 Chavagnat F, Casey MG, Meyer J. Purification, characterization, gene cloning, sequencing, and overexpression of aminopeptidase N from Streptococcus thermophilus A. Appl. Environ. Microbiol. 65, 3001-3007 (1999).

83 Matsui M, Fowler JH, Walling LL. Leucine aminopeptidases: diversity in structure and function. Biol. Chem. 387, 1535-1544 (2006).

84 Martin RE, Kirk K. Transport of the essential nutrient isoleucine in human erythrocytes infected with the malaria parasite Plasmodium falciparum. Blood 109, 2217-2224 (2007).

85 Liu J, Istvan ES, Gluzman IY, Gross J, Goldberg DE. Plasmodium falciparum ensures its amino acid supply with multiple acquisition pathways and redundant proteolytic enzyme systems. Proc. Natl Acad. Sci. USA 103, 8840-8845 (2006). 
86 Sherman IW. Amino acid metabolism and protein synthesis in malarial parasites. Bull. World Health Organ. 55, 265-276 (1977).

87 Nankya-Kitaka MF, Curley GP, Gavigan CS, Bell A, Dalton JP. Plasmodium chabaudi chabaudi and P. falciparum: inhibition of aminopeptidase and parasite growth by bestatin and nitrobestatin. Parasitol. Res. 84, 552-558 (1998).

88 Gavigan CS, Dalton JP, Bell A. The role of aminopeptidases in haemoglobin degradation in Plasmodium falciparuminfected erythrocytes. Mol. Biochem. Parasitol. 117, 37-48 (2001).

89 Skinner-Adams TS, Lowther J, Teuscher F et al. Identification of phosphinate dipeptide analog inhibitors directed against the Plasmodium falciparum M17 leucine aminopeptidase as lead antimalarial compounds. J. Med. Chem. 50, 6024-6031 (2007).

- Identifies the neutral aminopeptidases as antimalaria drug targets.

90 Maric S, Donnelly SM, Robinson MW et al. The M17 leucine aminopeptidase of the malaria parasite Plasmodium falciparum: importance of active site metal ions in the binding of substrates and inhibitors. Biochemistry 48(23), 5435-5439 (2009)

91 Gardiner DL, Trenholme KR, SkinnerAdams TS, Stack CM, Dalton JP. Overexpression of leucyl aminopeptidase in Plasmodium falciparum parasites. Target for the antimalarial activity of bestatin. J. Biol. Chem. 281, 1741-1745 (2006).

- Describes the use of transgenic overexpression to validate a drug target in $P$. falciparum.

92 Basten DE, Moers AP, Ooyen AJ, Schaap PJ. Characterisation of Aspergillus niger prolyl aminopeptidase. Mol. Genet. Genomics 272(6), 673-679 (2005).

93 Wilk S, Wilk E, Magnusson RP. Purification, characterisation and cloning of a cytosolic aspartyl aminopeptidase. J. Biol. Chem. 273, 15961-15970 (1998).

94 Yokoyama R, Kawasaki H, Hirano H. Identification of yeast aspartyl aminopeptidase gene by purifying and characterizing its product from yeast cells. FEBS J. 273(1), 192-198 (2006).

95 Teuscher F, Lowther J, Skinner-Adams TS et al. The M18 aspartyl aminopeptidase of the human malaria parasite Plasmodium falciparum. J. Biol. Chem. 282(42), 30817-30826 (2007).

96 Lauterbach SB, Lanzillotti R, Coetzer TL. Construction and use of Plasmodium falciparum phage display libraries to identify host parasite interactions. Malar. J. 2(1), 47 (2003).

97 Marti M, Good RT, Rug M, Knuepfer E, Cowman AF. Targeting malaria virulence and remodeling proteins to the host erythrocyte. Science 306(5703), 1930-1933 (2004).

98 Hiller NL, Bhattacharjee S, van Ooij C et al. A host targeting signal in virulence proteins reveals a secretome in malarial infection. Science 306(5703), 1934-1937 (2004).

\section{Websites}

101 World Malaria report http://apps.who.int/malaria/wmr2008

- Gives an overall picture of malaria today, along with the now positive outcomes that intervention is achieving in Africa.

102 WHO. Guidelines for the Treatment of Malaria. World Health Organization, Geneva, Switzerland (2006) http://apps.who.int/malaria/docs/ TreatmentGuidelines2006.pdf

103 PlasmoDB. Plasmodium genome resource www.plasmodb.org/plasmo/home.jsp

$104 \mathrm{WHO} / \mathrm{TDR}$ malaria database www.wehi.edu.au/MalDB-www/who.html

105 RCSB PDB Protein data bank. A resource for studying biological macromolecules www.pdb.org

106 Min T, Shapiro L. Crystal structure of aminopeptidase (M18 family) from Thermotoga maritim. RCSB Protein Database Reference 2GLF (2006) www.rcsb.org/pdb/explore. do? structureId=2GLF

\section{Affiliations}

- Donald L Gardiner Malaria Biology Laboratory, Queensland Institute of Medical Research, 300 Herston Road, Herston, QLD 4006, Australia

Tel.: +61 733620432

Fax: +61 733620104

don.gardiner@qimr.edu.au
- $\quad$ Tina S Skinner-Adams

Malaria Biology Laboratory, Queensland Institute of Medical Research, 300 Herston Road, Herston, QLD 4006, Australia

- Christopher L Brown

School of Biomolecular and Physical Science, Griffith University, Nathan, QLD 4111, Australia and

Eskitis Institute for Cell and Molecular Therapies, Griffith University, Nathan, Australia

- Katherine T Andrews Griffith Medical Research College, a joint program of Griffith University and the Queensland Institute of Medical Research, Griffith University, Nathan, Australia and

Eskitis Institute for Cell and Molecular Therapies, Griffith University, Nathan, Australia

and

Clinical Tropical Medicine Laboratory, Queensland Institute of Medical Research, 300 Herston Road, Herston, QLD 4006, Australia

- Colin M Stack School of Biomedical Health, Department of Medical Microbiology, University of Western Sydney, Narellan Road, Campbelltown, NSW 2560, Australia

- James S McCarthy Central Medical Division, School of Medicine, University of Queensland, St Lucia, Queensland, Australia and

Clinical Tropical Medicine Laboratory, Queensland Institute of Medical Research, 300 Herston Road, Herston, QLD 4006, Australia

- John P Dalton

Institute of Parasitology, McGill University, 21111 Lakeshore Road, Sainte Anne de Bellevue, QC H9X 3V9, Canada

- Katharine R Trenholme

Malaria Biology Laboratory, Queensland Institute of Medical Research, 300 Herston Road, Herston, QLD 4006, Australia 